STUDI

FRANCESI

\section{Studi Francesi}

Rivista quadrimestrale fondata da Franco Simone

159 (LIII | III) | 2009

Varia

\title{
Anne-Laure Metzger-Rambach, «Le texte emprunté». Étude comparée du "Narrenschiff" de Sebastian Brant et de ses adaptations (1494-1509)
}

\section{Olga Anna Duhl}

\section{(2) OpenEdition \\ Journals}

Édition électronique

URL : http://journals.openedition.org/studifrancesi/7499

DOI : 10.4000/studifrancesi.7499

ISSN : 2421-5856

Éditeur

Rosenberg \& Sellier

\section{Édition imprimée}

Date de publication : 1 décembre 2009

Pagination : 607-608

ISSN : 0039-2944

\section{Référence électronique}

Olga Anna Duhl, «Anne-Laure Metzger-Rambach, "Le texte emprunté». Étude comparée du "Narrenschiff" de Sebastian Brant et de ses adaptations (1494-1509) ", Studi Francesi [En ligne], 159 (LIII | III) | 2009, mis en ligne le 30 novembre 2015, consulté le 09 janvier 2021. URL : http://journals.openedition.org/ studifrancesi/7499; DOI : https://doi.org/10.4000/studifrancesi.7499

Ce document a été généré automatiquement le 9 janvier 2021.

\section{cc) (†)}

Studi Francesi è distribuita con Licenza Creative Commons Attribuzione - Non commerciale - Non opere derivate 4.0 Internazionale. 


\title{
Anne-Laure Metzger-Rambach, «Le texte emprunté». Étude comparée du "Narrenschiff" de Sebastian Brant et de ses adaptations (1494-1509)
}

\author{
Olga Anna Duhl
}

\section{RÉFÉRENCE}

ANNE LAURE METZGER RAMBACH, «Le texte emprunté». Étude comparée du "Narrenschiff" de Sebastian Brant et de ses adaptations (1494-1509), Paris, Champion, 2008 («Études et Essais sur la Renaissance», LXXVI), pp. 450

1 Il faut féliciter Anne-Laure Metzger-Rambach pour offrir au lecteur une nouvelle voie d'accès au riche héritage plurilingue dont fut la source le Narrenschiff, «La Nef des fous», (Bâle, 1494), la célèbre satire des folies du monde de Sebastian Brant, entre la date de sa publication et la première décennie $d u x v I^{e}$ siècle. Certes, d'excellentes études consacrées aux divers aspects, culturels, littéraires, sociaux et linguistiques du Narrenschiff, ont soigneusement préparé le terrain. Mais c'est A.-L.M.-R. qui parvient, avec beaucoup de compétence et de finesse, à faire dialoguer entre elles les nombreuses versions, latines, françaises et anglaises, du Narrenschiff, dont elle procure également des traductions précises, et cela pour montrer que la traduction correspond à cette époque à une forme d'adaptation propice non seulement au contact des cultures et à l'enrichissement des langues vernaculaires, mais aussi à la création. Voilà les deux volets d'une argumentation menée avec soin à la lumière des théories de la traduction, classique et patristique, au fil des quatre parties, divisées à leur tour en plusieurs chapitres, qui structurent cette riche étude.

Dans son introduction, A.-L.M.-R. s'attache à situer le Narrenschiff dans la tradition littéraire de la facétie qui connaît un nouvel éclat à partir de la fin du $\mathrm{xv}^{\mathrm{e}}$ siècle, auquel 
Brant apporta sa propre contribution. En ce qui concerne le «motif des fous embarqués à bord d'un navire» (p. 13), il ne s'agit guère d'une invention de Brant, même si son succès fut par la suite intimement lié au nom de ce dernier, mais d'une forme de satire qui se développe au début de l'ère moderne. Un premier aperçu des éditions, traductions, nouvelles compositions et études critiques dont fut la source le Narrenschiff, y compris lors du $500^{\mathrm{e}}$ anniversaire de sa publication, permet d'apprécier l'envergure de son succès et l'actualité du présent travail de synthèse. Quant aux mobiles de ce succès, A.-L.M.-R. évoque notamment la présence, au sein des éditions des Nefs, des gravures, dont certaines furent attribuées à Albrecht Dürer, et surtout la disponibilité, à partir de 1497, d'une traduction latine, la Stultifera navis, réalisée par Jacob Locher, disciple de Brant. C'est en effet cette version qui s'imposa, grâce à la supériorité stylistique du latin, comme modèle de la plupart des traductions vernaculaires, à l'exception des traductions anglaises qui se fondent en partie sur des sources françaises.

3 La première partie s'ouvre sur la «présentation du corpus»: en dehors d'un aperçu des éditions du Narrenschiff, dont l'histoire est bien connue, A.-L.M.-R. dresse une liste complète des traductions et des adaptations latines (Jacob Locher, Josse Bade), françaises (Pierre Rivière, Jean Drouyn et l'Anonyme de Marnef), anglaises (Henry Watson et Alexander Barclay), et flamande, qu'elle fait suivre des notices biographiques des auteurs, y compris les Frères de Marnef, imprimeurs parisiens dont la contribution à la diffusion des Nefs est mise à juste titre en relief. Il manque cependant des indications permettant d'identifier dès le début les éditions utilisées, à l'exception du Narrenschiff (n. 1 p. 11). Des réflexions théoriques sur le rapport entre langue dominante et langues dominées qu'entretiennent, respectivement, le latin et les langues vulgaires, font ressortir la contribution de l'héritage textuel du Narrenschiff au développement de ces dernières, même si le latin joue toujours un rôle dans ce processus, et qu'une "généalogie» des nefs des fous (p. 42) illustre de manière utile. L'analyse des textes liminaires permet ensuite de cerner les lignes directrices du programme à double détente des translateurs: d'une part, se conformer à l'idéologie du texte source; d'autre part, prendre en compte les particularités des langues cibles sans oublier les (in)compétences supposées ou réelles des lecteurs. Mais A.-L.M.-R. souligne également le rôle de la nef et du fou à véhiculer les sens allégoriques du Narrenschiff, susceptibles d'imposer des limites à la créativité du traducteur (sauf pour Josse Bade qui ne cesse d'affirmer son talent, par exemple, en féminisant et en multipliant les nefs), et de faire ressortir le "caractère éminemment moral de la démarche d'écriture» (p. 109). Néanmoins, malgré leur dette envers le texte latin de Locher, et c'est là l'un des points essentiels de cette étude, les traductions présentent des qualités révélatrices de «la capacité» que possède la langue vernaculaire «de pouvoir exister hors de toute sujétion à la langue source» (p. 122). Des considérations théoriques sur la traduction «selon le sens» pratiquée par les translateurs, qui dérive de la «tradition patristique» (p. 122), permettent d'appréhender les enjeux de la création qu'illustrent les translateurs individuels.

4 La deuxième partie est consacrée aux techniques de la traduction (calque, réduplication synonymique), dont l'analyse permet d'apprécier, grâce à des tableaux comparatifs fort éclairants (pp. 140, 147, 150, 152-154), les capacités expressives des langues vulgaires, y compris sous la plume des traducteurs. En revanche, le commentaire se révèle comme une technique d'explication de texte susceptible de faire ressortir non seulement le statut autoritaire d'une source comme la Bible, mais aussi l'esprit créateur du 
translateur (Josse Bade offre encore une fois un bon exemple) qui devient «auteur en second» (p. 213), pour reprendre l'heureuse formule d'A.-L.M.-R.

Dans la troisième partie, A.-L.M.-R. nous livre son analyse des techniques et des sens de la «création» et de l'«appropriation» (p. 223). Par exemple, Locher préfère une «forme de traduction sélective» (p. 225) qui consiste en plusieurs modifications affectant le texte source, n'ayant d'autre but que de prêter à celui-ci une allure esthétique inspirée du latin, au prix même de renoncer à ses meilleurs effets satiriques. Chez Jehan Drouyn, qui traduit de rime en prose au sein de la même langue, l'appropriation s'effectue en revanche au profit d'un modèle de brièveté et de clarté. Quant aux sens de l'appropriation, il s'agit pour les traducteurs de faire valoir la «dimension nationale» de l'écriture (p. 247) (ne devrait-on pas parler plutôt de «protonationalisme» à la p. 66?), qui transparaît dans les passages traitant de l'avancée de l'Empire ottoman en Europe: le discours moral sert alors de moyen d'exhortation politique qui se module en fonction des allégeances individuelles des translateurs. Néanmoins, c'est la conversion des fous, non pas la visée politique des adaptateurs qui s'impose comme le fil conducteur des œuvres, souligne A.-L.M.-R. par le biais d'une analyse des moyens susceptibles de tirer le discours moral vers la prédication (exempla, oralité).

Dans la quatrième partie, il s'agit des «éléments paratextuels» (p. 307) qui servent de véhicules pour assurer la «continuité entre les Nefs» (p. 332). A.-L.M.-R. se penche d'abord sur l'ordre des chapitres, repris à quelques modifications près d'un texte à l'autre, qui reflète le souci de Brant de faire aboutir le discours moral à la doctrine du salut. Elle évoque ensuite la présence des gravures au sein des adaptations de la Nef, dont certains motifs renvoient au genre didactique du «miroir» invitant le lecteur à s'exercer à l'introspection (p. 330). Sont enfin examinées les origines classiques et bibliques du motif de la nef des fous, repris par les Pères de l'Eglise chez qui le Christ devient le commandant du vaisseau, mais que Brant interprète à sa manière en privant les fous d'une telle présence salutaire. Le motif de la nef acquiert en revanche une valeur plus concrète chez les translateurs qui n'oublient pas qu'à cette époque la navigation est une forme d'errance vouée à l'échec et symbolique du péché. Si la conversion devient une possibilité chez Drouyn, c'est parce qu'il sait transformer la nef en «un lieu de prédication» (p. 375).

Dans les conclusions, l'A. revient sur l'importance de la version latine dans la diffusion des Nefs et insiste sur la volonté des traducteurs de s'affirmer comme auteurs mais dont les voix restent tributaires du projet didactique de Brant.

Quatre annexes illustrant les concordances ou les écarts entre les différentes versions viennent compléter l'étude. La bibliographie est substantielle malgré certains silences (il manque, par exemple, des références aux nouvelles recherches consacrées à La nef des folles, ou encore aux études fondamentales d'Alexandre Lorian sur la prose et de Jacques Monfrin sur la traduction). Mais cela (non plus que les coquilles) n'ôte rien à l'intérêt de ce livre qui apporte une contribution précieuse à nos connaissances de l'impact du Narrenschiff sur les cultures vernaculaires qui profitent de l'exercice d'une forme de traduction libre, destinée pourtant à céder le pas à une pratique plus rigoureuse car fidèle à la lettre, mais sans doute moins favorable à l'affirmation de la voix créatrice. 